\title{
Dental erosion: In vitro model of wine assessor's erosion
}

\author{
Tong Bee Mok, ${ }^{*}$ J McIntyre, ${ }^{*}$ D Hunt*
}

\begin{abstract}
Background: Wine makers and assessors frequently experience severe dental erosion. The objectives of this study were to develop an in vitro model of dental erosion caused by frequent wine contact with teeth, and to use this model to assess the effectiveness of a variety of methods which might protect against this form of erosion.

Methods: An initial pilot study found that riesling style wine was more erosive than champagne style, and both more than claret. Wine tasting was simulated by subjecting exposed windows of enamel and root surfaces on 50 intact, extracted human teeth to 1400 one minute exposures to white wine ( $\mathrm{pH}$ 3.2). A variety of dental materials were applied to the exposed windows on groups of teeth prior to erosive challenge, to assess their protective ability. Results: Protective resin coatings and fluoride varnishes protected both enamel and roots against wine induced erosion. A high degree of protection was provided by APF gel, with less by $\mathrm{NaF}$ gel.

Conclusions: It was concluded that dentists may be able to help minimise erosion resulting from frequent wine-tasting in their patients by the clinical application of one or a combination of these agents at times prior to prolonged assessment periods.
\end{abstract}

Key words: Erosion, wine, protection, resins, dentine bonding agents, fluorides.

(Received for publication March 1997. Revised September 1999. Accepted November 1999.)

\section{INTRODUCTION}

Dental erosion, when caused by frequent contact of wine with teeth, can involve severe loss of tooth enamel and a high level of dentinal hypersensitivity. The types of erosive damage may vary considerably in different people and will frequently involve incisal and cuspal tip enamel, loss of enamel from both lingual and labial surfaces of maxillary anterior teeth and severe loss of structure around the labial cemento-enamel junction of both maxillary and mandibular anteriors and premolars. Loss of cuspal enamel may accentuate further wear through abrasion and attrition. Cervical erosion frequently leads to accentuated wear through abrasion and abfraction (Fig 1,2).

*Department of Dentistry, The University of Adelaide.
At greatest risk of dental erosion are wine judges, who may taste up to 200 wines a day for four consecutive days several times a year, and winemakers. As the $\mathrm{pH}$ of wines is usually around 3-3.5, such a level of acidity in contact with the teeth so frequently for these long periods is sufficient to overwhelm the normal levels of protection of saliva, pellicle and plaque and cause rapid demineralisation.

It is crucial that the dental profession assist those patients involved in the wine industry or amateur winetasters, by providing both protection and advice as to how erosion can be minimised. However, before this can occur, the nature of wine erosion needs to be fully explored and the effectiveness of various methods of protection needs to be analysed, initially in the laboratory.

The potential for wines to cause erosion results from their fruit acid content, of which tartaric acid is the most abundant. However, there is no correlation between the $\mathrm{pH}$ and the depth of enamel dissolved. ${ }^{1}$ Erosion is found to be a function of the acid dissociation constant, the calculated unionised acid concentration $^{2}$ and the concentration of demineralisation inhibiting agents. ${ }^{3}$ Thus, in demineralisation processes, the acid type and concentration are more important than the $\mathrm{pH}$ alone. Due to the fact that different wines have different combinations and concentrations of acid types and other inhibiting factors present, the erosive potential varies.

Winemakers believe that champagne-style wines are usually the most damaging to teeth, with red wines least damaging in terms of erosion. The longer maturation periods required for red wines may result in the greater production of potential inhibitors of erosion. Whether this is so, and the nature of such inhibitors, needs further investigation. Red wines are known to cause considerable problems with severe staining of teeth for both tasters and frequent consumers.

The aim of this study was to establish an in vitro model of wine erosion and use this model to test the effectiveness of various protective methods against this form of erosion. Imfeld ${ }^{4}$ has proposed that the key components of a preventive program are to diminish the frequency and severity of the acidic challenge; enhance salivary flow; provide chemical protection; 


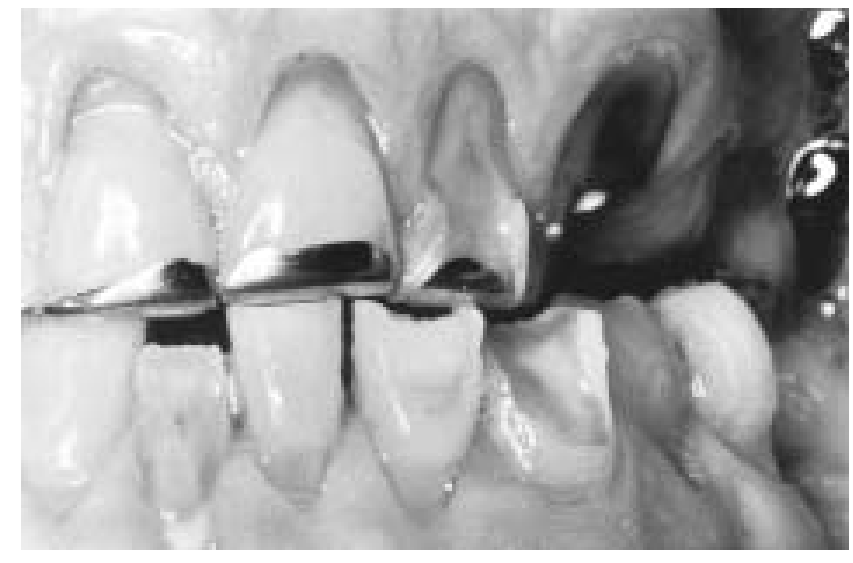

Fig 1. Erosion of enamel from frequent winetasting. Note the restoration standing proud above the eroded enamel.

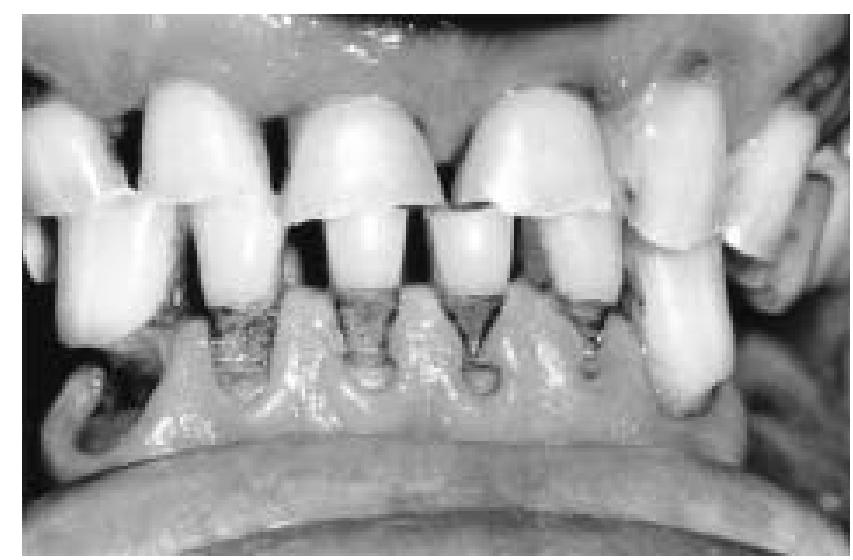

Fig 2. Erosion contributing to severe root abrasion and loss of incisal length in a wine assessor. In this case, erosion has accentuated the severity of wear through abrasion and attrition.

minimise abrasion; enhance tooth resistance; or offer mechanical protection. Enhancing tooth resistance and offering mechanical protection are likely to be the most successful methods to treat wine assessors or frequent consumers. Those most at risk of erosion, wine assessors and winemakers, are required to taste wine frequently. The entry of wine into the mouth stimulates salivary flow. However, different individuals have differing levels of salivary protection, which are difficult to modify. Even so, further investigation is needed to find ways of maximising the protective roles of saliva, pellicle and plaque. Without risking alteration to the taste, chemical protection cannot be provided within the wine itself. Hence, in this study, fluoride gels and varnishes were tested for their ability to provide increased protection against demineralisation, and sealants were tested for their ability to provide protection using this model.

\section{MATERIALS AND METHODS \\ Wine selection}

White wine is generally regarded as being more erosive than red, with champagne-style wines usually the most erosive. As the wine might preferably be used at room temperature, a pilot investigation was carried out to determine which was the most erosive under these conditions. Forty-two intact extracted premolars were cleaned of all soft and hard debris and washed and stored in distilled water to which was added thymol ( 0.1 per cent final concentration thymol/ddw) until required for testing. The premolars were then painted with water-resistant varnish, leaving experimental windows exposed on crowns $(2 \times 2 \mathrm{~mm})$ and on the root surface commencing at least $1 \mathrm{~mm}$ apical to the labial or lingual aspect of the cementoenamel junction $(1 \times 4 \mathrm{~mm})$. Twenty-one tooth crowns were placed (seven each) in $100 \mathrm{ml}$ samples of champagne-style wine ( $\mathrm{pH} 3.3$ ), white wine (rieslingstyle, pH3.2) or red wine (claret, $\mathrm{pH} 3.2$ ) at bench temperature (room temperature controlled to $25^{\circ} \mathrm{C}$ daily). A second group of 21 teeth was placed in similar volumes of those wines and incubated continuously at $37^{\circ} \mathrm{C}$. Depths of erosion were measured after daily continuous exposure, one sample being removed for sectioning each day in each category.

The teeth were sectioned by using a diamond blade on a hard tissue sectioning machine. Initially, crowns were separated from roots by a transverse cut through the most apical aspect of the cemento-enamel junction. Crowns were sectioned longitudinally buccolingually through the centre of the erosion lesion. Roots had sections approximately $150 \mu \mathrm{m}$ thick cut longitudinally buccolingually through the demineralised lesions. All sections were kept in thymol/ddw solution to avoid microbial contamination until examination. The depths of erosion were then measured by stereoscopic microscopy, using an optical graduated scale for enamel, and then by transmission microscopy for roots. The eyepiece graduated scale had been previously calibrated against a graduated metric standard.

For crowns, there appeared to be little difference in depths of erosion resulting from exposure to white wine or champagne-style wine, both at room temperature and at $37^{\circ} \mathrm{C}$. Erosion resulting from red wine contact was substantially less than both white wines (Fig 3, 4). These results will be described in detail later.

White wine, used at room temperature, was chosen to be the erosive test agent in establishing the model of wine erosion.

\section{Model development}

Tooth samples

Fifty premolar crowns with intact adjacent root cementum surfaces were collected and cleaned. The teeth were then painted with varnish, leaving windows of equivalent size on both enamel and dentine (Fig 5). Using sticky wax, the teeth were then adhered to thin wooden sticks and the sticks attached to a polystyrene sheet so all teeth could be simultaneously submerged in a bath of wine. The tooth specimens were placed in 10 rows of five teeth each.

\section{Enamel and root surface pretreatment categories}

The teeth were subjected to 10 categories of pretreatment, five teeth representing each group (Table 1). 


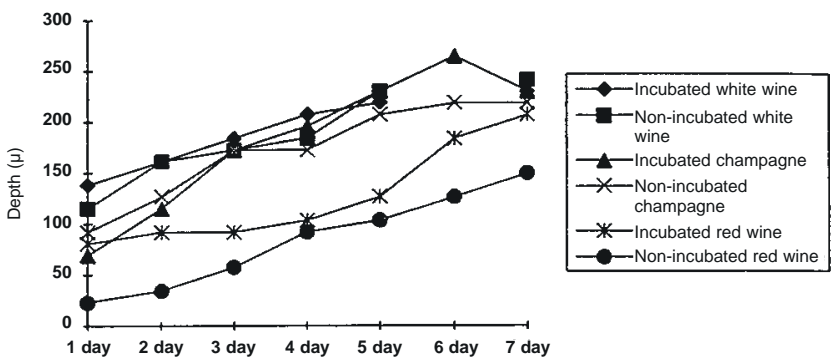

Fig 3. Depth of erosion in enamel $(\mu \mathrm{m})$ from continuous exposure to wine for up to seven days, incubated at $37^{\circ} \mathrm{C}$ and at room temperature.
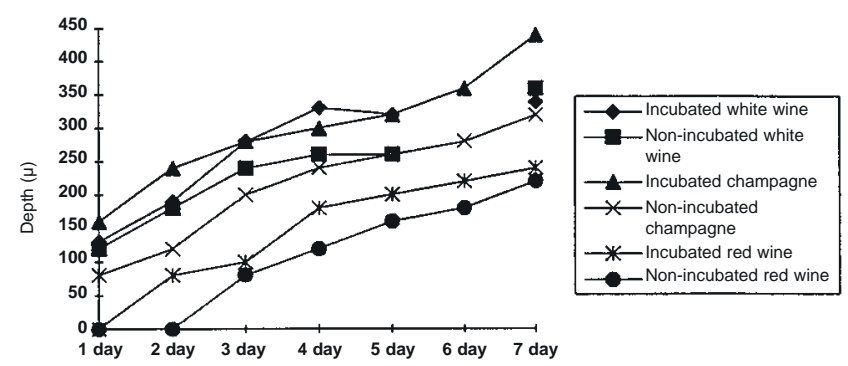

Fig 4. Depth of erosion in cementum/dentine $(\mu \mathrm{m})$ from continuous exposure to wine for up to seven days, incubated at $37^{\circ} \mathrm{C}$ and at room temperature.

\section{Erosion simulation method}

As wine assessors taste wines by holding them in their mouths for 30-60 seconds, then expectorate and rinse with water before tasting the next wine, an attempt was made to simulate this process as closely as possible. A cyclic dipping system was established, requiring baths of wine and $\mathrm{ddw}$, close to a jet of compressed air to permit drying of specimens prior to further exposure. The teeth were dipped in the wine bath at room temperature for multiple one-minute time increments, followed by rinsing in distilled water for one minute and drying for another minute. The wine and distilled water were renewed after every 50 exposures to ensure the wine did not become saturated with calcium and phosphate ions.

Enamel surfaces were observed closely to assess when the depth of enamel erosion in the unprotected samples of teeth was visually discernable. The experiment was concluded after 1,400 minutes of accumulated exposure (simulating nearly 24 hours of intermittent erosion).

\section{RESULTS}

\section{Continuous erosion pilot study}

Continuous exposure of both enamel and root surface cementum to samples of riesling- and champagne-style wines and claret for up to seven days (10,080 minutes) both at room temperature $\left(25^{\circ} \mathrm{C}\right)$ and at $37^{\circ} \mathrm{C}($ Fig 3,4$)$ showed red wine was the least erosive, resulting in $145 \mu \mathrm{m}$ depth of erosion when not incubated and $200 \mu \mathrm{m}$ when incubated. The white wine resulted in the deepest erosion $(225 \mu \mathrm{m}$ whether incubated or not). Champagne-style wine, which is usually the most erosive of wines, in this case resulted in the same level of erosion as white wine. Even though the data are

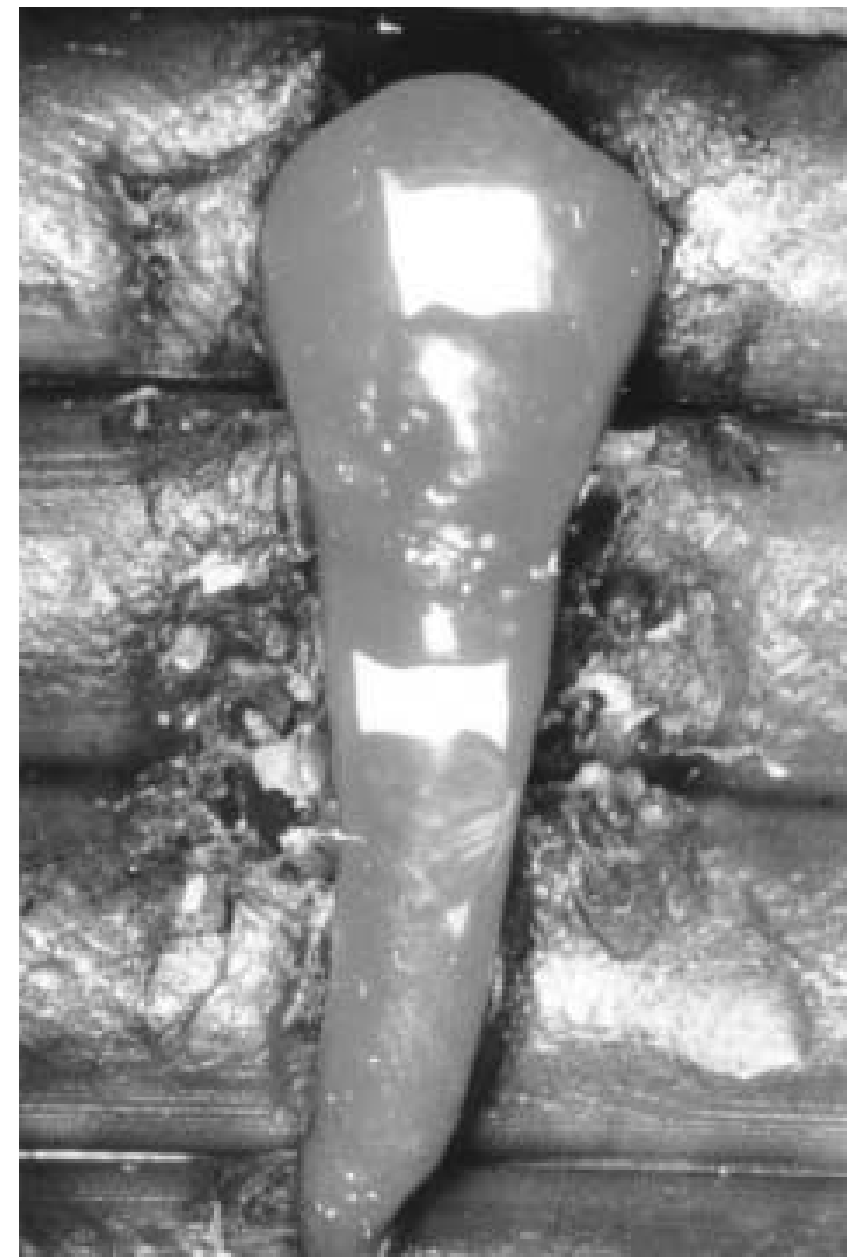

Fig 5. Whole tooth covered with varnish, leaving windows of equivalent dimension exposed on both enamel and root surface.

derived from one sample a day, there appears to be a steady rate of increase in depth in erosion following a substantial rate of progression for white wines during the first day of exposure. This rate of progress appears to be similar for both enamel and cementum/dentine, though with overall greater erosion experienced in the latter. However, there was some delay before claret began to cause root surface damage.

\section{White wine: Model of wine erosion}

Table 2 shows that, without protection, a mean of $67.7 \mu \mathrm{m}$ of erosion resulted for the five unprotected windows in enamel following 1,400 minutes of wine exposure. In root cementum/dentine, erosion to a depth of $177.5 \mu \mathrm{m}$ resulted (Table 3 ).

\section{Protective potential of various materials}

No erosion was observed (Table 2, Fig 6) in crowns which were coated with fissure sealant, Duraphat and Fluor Protector. A comparison between groups C, D, E and $\mathrm{F}$ (pretreatment with APF fluoride gel) (Table 1), showed group D had the most erosion. Thus, as might be expected, the more frequently APF gel is applied to crowns, the more protective it becomes. On a comparative basis, $\mathrm{NaF}$ gel could only provide slight erosion protection. 
Table 1. Enamel and root surface pretreatment categories.

\begin{tabular}{|c|c|}
\hline Category & Treatment \\
\hline A & Control unprotected. \\
\hline B & $\begin{array}{l}\text { A coating of light cured Delton LC Pit and Fissure Sealant (Johnson \& Johnson, US) was placed over enamel windows following } \\
\text { acid etching with } 37 \text { per cent phosphoric acid for } 20 \text { seconds; a single coating of Scotchbond Multipurpose dentine bonding agent } \\
\text { ( } 3 \mathrm{M} \text {, US) was placed over root surface windows on the same category of teeth following conditioning with } 10 \text { per cent polyacrylic } \\
\text { acid for } 20 \text { seconds. Both enamel and root surface windows were washed with a jet of ddw for } 10 \text { seconds prior to the erosive } \\
\text { challenge. }\end{array}$ \\
\hline $\mathrm{C}$ & $\begin{array}{l}\text { Both windows were covered with excess } 1.23 \text { per cent acidulated phosphate fluoride (APF) Colgate Orofluor Gel (Colgate, } \\
\text { Australia) placed for five minutes and then washed thoroughly with ddw for } 20 \text { seconds to remove the gel before every } 100 \\
\text { exposures. }\end{array}$ \\
\hline $\mathrm{D}$ & APF gel was placed as above for five minutes and then removed by washing with ddw before every 40 exposures. \\
\hline E & APF gel was placed as above for five minutes and then removed by washing with ddw before every 20 exposures. \\
\hline $\mathrm{F}$ & APF gel was placed for five minutes and then washed with ddw before every 10 exposures. \\
\hline G & $\begin{array}{l}\text { Excess Duraphat fluoride varnish (Inpharma, Germany) was placed over both windows for } 20 \text { minutes before every } 100 \\
\text { exposures and permitted to remain on the surface during wine exposure. }\end{array}$ \\
\hline $\mathrm{H}$ & $\begin{array}{l}\text { A single layer of Fluor Protector fluoride varnish (Vivadent, Liechtenstein) was placed over both windows for } 20 \text { minutes before } \\
\text { every } 100 \text { exposures and not washed prior to wine exposure. }\end{array}$ \\
\hline I & $\begin{array}{l}\text { Excess sodium fluoride ( } \mathrm{NaF} 2.2 \text { per cent) gel was placed over both windows for five minutes and then washed away with a jet } \\
\text { of ddw for } 20 \text { seconds before every } 100 \text { exposures. }\end{array}$ \\
\hline $\mathrm{J}$ & $\mathrm{NaF}$ gel was placed as above for five minutes and then washed away with ddw before every 40 exposures. \\
\hline
\end{tabular}

The results for groups B, C, D, E, F and $\mathrm{H}$ in root sections showed complete protection had been achieved by the protective measures used (Table 3, Fig 7). The frequency with which APF gel is applied to root surfaces did not appear to be critical. Again, $\mathrm{NaF}$ gel was not able to provide the same degree of protection as APF gel (I and J).

For some root surfaces, Duraphat showed no protection at all whereas, for others, maximum protection was achieved. It was noted the layer of Duraphat had been dislodged by the action of dipping and drying on some root surfaces, though not on enamel. This would explain the wide variation in results, though why Duraphat is less able to adhere to root surfaces than enamel is not known.

\section{DISCUSSION}

In vitro models of endogenous erosion have been used to determine the rate of erosion of enamel, cementum and dentine caused by a wide variety of known erosive substances (citric acid, acetic acid and hydrochloric acid). ${ }^{5-6}$ These model systems have also been used to test the effectiveness of prior applications of APF gel in providing protection against simulated gastric acid. ${ }^{7}$ In this study, similar in vitro methods were used to model wine assessor's erosion and test the effectiveness of potential inhibitors of this form of erosion.

\section{Table 2. Mean depth of enamel lost by erosion} in $\mu \mathrm{m}$ (SD).

\begin{tabular}{llcc}
\hline A & Unprotected (control) & 67.7 & $(8.6)$ \\
B & Fissure sealant & - & - \\
C & APF gel before 100 exp & 18.4 & $(4.8)$ \\
D & APF gel before 40 exp & 20.7 & $(16.6)$ \\
E & APF gel before 20 exp & 12.7 & $(2.3)$ \\
F & APF gel before 10 exp & 2.3 & $(0.6)$ \\
G & Duraphat & - & - \\
H & Fluor Protector & - & - \\
I & NaF gel before 100 exp & 42.6 & $(18.0)$ \\
J & NaF gel before 40 exp & 42.6 & $(5.1)$ \\
\hline
\end{tabular}

As wine judges and winemakers are most at risk of wine erosion, it was considered important to attempt to simulate the contact of wine with teeth in this in vitro model. Hence, the decision to use the incremental oneminute exposures, though extremely laborious, was considered important. In wine-tasting, a sip of wine is left in the mouth for 30-60 seconds and kept moving around the mouth, being kept for some time near the anterior taste buds in the tongue.

The most frequent complaint presented to dentists by professional wine assessors is cervical hypersensitivity. In these cases, dentists frequently detect erosion of enamel or aggressive abrasion or attrition exposing dentine to the erosive acids. For this reason, erosion was measured in both enamel and root cementum/ dentine. In the initial pilot study, white wines were shown to be far more aggressive in eroding cementum/ dentine than the action of red wines in eroding enamel. It took two days of exposure of roots to red wine at room temperature before any erosion of root cementum could be recorded. Why sensitivity of enamel to red wine erosion is greater than that of root cementum is not known.

The results of the pilot study were surprising in that from anecdotal evidence from wine assessors it was expected champagne-style wines would have been significantly more erosive than riesling-style wines. However, very dry riesling-style wines may be expected

Table 3. Mean depth of root cementum/dentine lost by erosion in $\mu \mathrm{m}$ (SD).

\begin{tabular}{llcc}
\hline A & Unprotected (control) & 177.5 & $(36.9)$ \\
B & Scotchbond Multipurpose & - & - \\
C & APF gel before 100 exp & - & - \\
D & APF gel before 40 exp & - & - \\
E & APF gel before 20 exp & - & - \\
F & APF gel before 10 exp & - & - \\
G & Duraphat & 44.0 & $(60.7)$ \\
H & Fluor Protector & - & - \\
I & NaF gel before 100 exp & 46.6 & $(12.8)$ \\
J & NaF gel before 40 exp & 135.3 & $(30.9)$ \\
\hline
\end{tabular}

Australian Dental Journal 2001;46:4. 


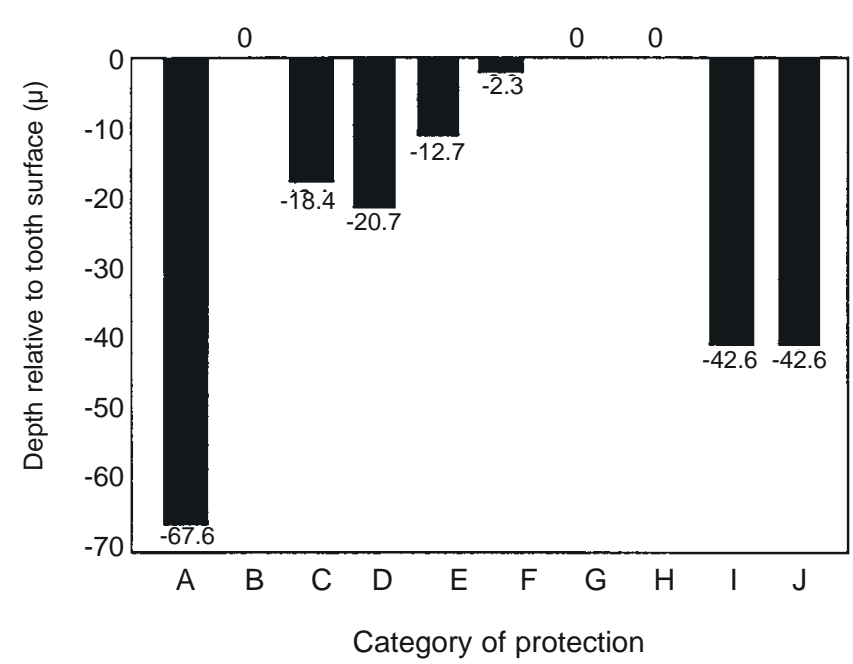

Fig 6. Depth of erosion in enamel $(\mu \mathrm{m})$ after 1,400 minutes exposure to wine, with varying categories of protection. A: Unprotected (control); B: Fissure sealant; C: APF gel before 100 exp; D: APF gel before 40 exp; E: APF gel before 20 exp; F: APF gel before 10 exp; G: Duraphat; H: Fluor Protector; I: NaF gel before 100 exp; J: NaF gel before 40 exp.

to be more acidic and, potentially, erosive. As there was little difference in the erosion caused by riesling- and champagne-style wines on both enamel and root cementum at both temperatures, it was decided to use the riesling-style wine, which is probably more widely consumed. It was also decided to carry out the tests at room temperature as is the practice of wine assessors, even though white wines are normally served chilled.

Wine assessors are careful not to ingest or apply anything which will alter their taste perception for a few hours prior to tasting. Hence, any protective method must be applicable some hours before tasting begins. The resin based protective methods were applicable the day before wine-tasting, as were use of APF and neutral NaF gels. The use of Duraphat and Fluor-Protector Varnish might be considered the morning of a tasting, though their effect on taste sensation would need to be thoroughly explored.

Interestingly, 1.23 per cent APF gel was found to be more effective against erosion in root surfaces than in crowns. Root surface sensitivity is a major problem for wine assessors. These surfaces are more rapidly eroded, increasing the risk that the remaining leathery collagen might be abraded by brushing before remineralisation can occur. The finding that 2 per cent $\mathrm{NaF}$ gel was not as effective as APF gel was not surprising. This finding is in accordance with the result shown by Kutler et $\mathrm{al}^{8}$ and Fung and McIntyre ${ }^{7}$ where multiple applications of $\mathrm{NaF}$ could only provide slight reduction in demineralisation. Presumably, this reduction results from the potential for increased concentrations of fluoride uptake into both enamel and dentine from the acidulated product, these increased concentrations providing prolonged protection against more acidic challenges.

The implications of these findings for the dental profession are significant. In routine wine-tasting competitions, where at least $80-100$ wines are tasted daily several days in a row, the use of resin protection

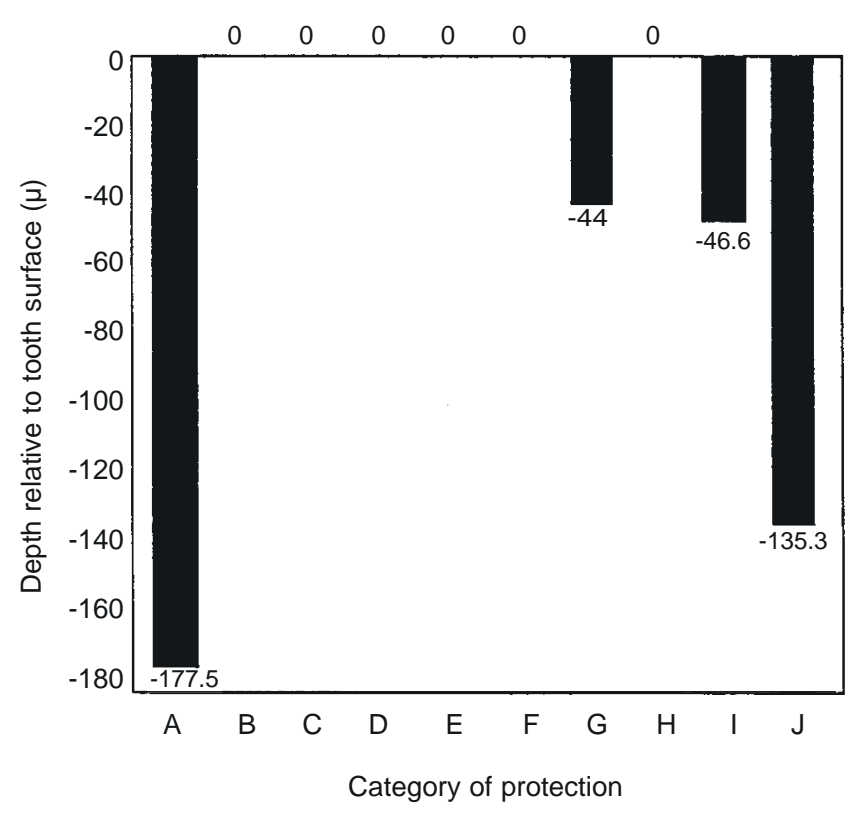

Fig 7. Depth of erosion on root cementum/dentine $(\mu \mathrm{m})$ after 1,400 minutes exposure to wine, with varying categories of protection. A: Unprotected (control); B: Scotchbond Multipurpose; C: APF gel before 100 exp; D: APF gel before 40 exp; E: APF gel before 20 exp; F: APF gel before 10 exp; G: Duraphat; H: Fluor Protector; I: NaF gel before 100 exp; J: NaF gel before 40 exp.

for vulnerable enamel and root surfaces might be the most certain erosion minimisation method. Also, the application of APF gels the evening prior to tasting has been shown to be most useful in slowing down the extent of erosive damage, particularly where dentine or cementum are exposed. Of the fluoride varnishes, Fluor Protector, due to its polyurethane coating, has the advantage of firmer binding and protection of the tooth surface, even though the fluoride concentration is now low (1,000ppm). However, due to varnish solvent ethyl acetate possibly affecting taste for some time following its application, the fluoride varnish would need to be applied professionally at least one day before a prolonged period of tasting. Duraphat, while having the advantage of being natural resins dissolved in alcohol and having a high fluoride concentration (23,600ppm), does nor bond closely to the teeth. However, Duraphat would prove to be useful if applied the day before a prolonged wine-tasting.

\section{CONCLUSION}

The present study has demonstrated there are several methods which may be effective against dental erosion caused by wine. Resin protection and Fluor Protector show great promise in providing protection to wine assessors and self-application of concentrated APF gel the evening prior to a tasting also appears to be useful. With increased awareness by both dentist and patient, the problem of wine-tasting induced dental pathology may be reduced.

\section{ACKNOWLEDGEMENTS}

The authors acknowledge the help and assistance rendered by Dr GJ Mount and the Australian Dental Research Foundation. 


\section{REFERENCES}

1. Wynn W, Haldi J. The erosive action of various fruit juices on the lower molar teeth of the albino rat. J Nutr 1948;35:489-497.

2. Featherstone JDB, Rogers BE. Effects of acetic, lactic and other organic acids on the formation of artificial carious lesions. Caries Res 1982;15:377-385.

3. Larsen MJ. Dissolution of enamel. Scand J Dent Res 1973;81:518522.

4. Imfeld T. Prevention of progression of dental erosion by professional and individual prophylactic measures. Eur J Oral Sci 1996;104:215-220.

5. Hunt D, McIntyre JM. The development of an in vitro model of dental erosion. J Dent Res 1992;71:985.
6. White I, McIntyre JM. The effect of artificial erosion on tooth root surfaces. J Dent Res 1992;71:986.

7. Fung L, McIntyre JM. Effectiveness of $1.23 \%$ APF gel in controlling enamel erosion in vitro. J Dent Res 1993;72:749.

8. Kutler B, Ireland RL. The effect of sodium fluoride application on dental caries experience in adults. J Dent Res 1953;32:458-462.

Address for correspondence/reprints: Dr JM McIntyre Department of Dentistry Adelaide University Adelaide, South Australia 5000 